\title{
Fluorescence quenching at high quencher concentrations
}

\author{
David Peak \\ Department of Physics, Union College, Schenectady, New York 12308
}

T. C. Werner and Richard M. Dennin, Jr.

Department of Chemistry, Union College, Schenectady, New York 12308

\author{
James K. Baird \\ Department of Chemistry, University of Alabama, Huntsville, Alabama 35899 \\ (Received 26 April 1983; accepted 22 June 1983)
}

\begin{abstract}
Chemical reactions occurring in dense media at high reactant concentrations can be described by rate "constants" which are actually functions of concentration. We present a theoretical model in which this socalled rate constant "renormalization" occurs for the specific case of fluorescence quenching in solution. We show that both the quenching and the excitation rate constants can become concentration dependent. We fit our theory to several sets of experimental data-our own and some from the literature-and show that excellent agreement is obtained by varying a single free parameter, namely, the efficiency with which a fluorophore-quencher collision leads to a quench of the excited state.
\end{abstract}

\section{INTRODUCTION}

When a bimolecular reaction of the type $A+B \rightarrow C$ occurs in a dense, inert solvent, the rate of formation of the product is bilinear in the concentrations of the reactants, after transient effects have vanished, as long as the reactant species are sufficiently dilute. In other words, under these conditions the rate of increase in the concentration of $\mathrm{C}$ can be represented by the expression $k[\mathrm{~A}][\mathrm{B}]$, where $k$ is a (second-order) rate constant and the quantities in square brackets are (molar) concentrations. At high reactant concentrations, however, the situation is more complex. A number of recent theoretical investigations of reactions at high concentration-while differing in quantitative detailarrive at the same qualitative conclusion: in dense solvents, where diffusion limits the rate of reactions, competition for each A molecule among all the B's, and for each $B$ among all the A's, leads to a "renormalization" of the bimolecular rate constant; the rate of formation of $\mathrm{C}$ at high reactant concentrations can be forced into a (pseudo-) bilinear form $k^{\prime}[\mathrm{A}][\mathrm{B}]$, but $k^{\prime}$ is then a function of $[A]$ and $[B] .^{1}$

Baird and Escott ${ }^{2}$ and, more recently, Keizer ${ }^{3}$ have argued that evidence for rate constant renormalization can be found in data from experiments on the quenching of fluorescence in liquids. In their view, the secondorder rate constant describing the collisional quench of an excited fluorophore by an impurity molecule in solution can acquire a quencher concentration dependence at high concentrations, because of the effects mentioned above; this, in turn, can cause the Stern-Volmer plots associated with such experiments to have positive curvature. When the theoretical models of these papers are fit to actual data, however, the fitting parameters, for some of the cases examined, take on physically unreasonable values.

Prior explanations for the origin of positively curved Stern-Volmer plots have been based on a variety of chemical and physical mechanisms, ${ }^{4}$ but, in essence, all of these have focused on the possible concentration dependence of the fluorescence excitation rate. It is worthy of note that these models also have associated fitting parameters whose values are sometimes difficult to interpret physically. In this paper, we show that competitive correlations between fluorophores and quenchers, when collisional quenching is diffusion controlled, actually lead to a renormalization of both the quenching and the excitation rate constants. When we compare the theoretical results derived here with experiment, we are able to obtain excellent agreement (using reasonable values for molecular variables) by adjusting a single fitting parameter: the quenching efficiency of a fluorophore-quencher collision.

In order to clarify these preliminary comments, we define, with some care, the specific system of interest. We consider fluorophores $A$ with a single excited state $A^{*}$ and impurity quencher molecules $Q$ which participate, in the presence of a dense, inert solvent, in the following reaction scheme:

$$
\begin{aligned}
& h \nu+\mathrm{A} \stackrel{k_{E}}{\longrightarrow} \mathrm{A}^{*}, \\
& \mathrm{~A} * \stackrel{k_{N R}}{\longrightarrow} \mathrm{A}, \\
& \mathrm{A}^{*} \stackrel{k_{F}}{\longrightarrow} \mathrm{A}+h v^{\prime}, \\
& \mathrm{A} *+\mathrm{Q} \stackrel{k_{\mathrm{Q}}}{\longrightarrow} \mathrm{A}+\mathrm{Q} .
\end{aligned}
$$

Reaction (1a) represents the excitation of $A^{*}$ by the absorption of a photon of frequency $u$; (1b) represents the nonradiative decay of $A^{*}$; (1c) represents fluorescent decay, leading to a photon of frequency $\nu^{\prime}$; and (1d) represents the collisional quench of $A^{*}$ by $Q$. If the exciting beam (the $\nu$-frequency photons) is of constant intensity the reaction scheme, (1a)-(1d), will eventually attain a steady state. We restrict our attention throughout this paper to this condition. The first-order rate 
constants $k_{E}, k_{N R}$, and $k_{F}$, and the second-order constant $k_{Q}$, are steady-state, infinite-dilution values. We will assume that the "intrinsic" processes (1b) and (1c) are unaffected by the presence of quencher. If we allow for the possibility that the character of excitation and collisional quenching might change (by whatever mechanism) as the concentration of $Q$ increases, we can then express the steady-state kinetics of the reaction system (1) by

$$
k_{E}^{\prime}[\mathrm{A}]=\left(k_{F}+k_{N R}+k_{Q}^{\prime}[\mathrm{Q}]\right)\left[\mathrm{A}^{*}\right] .
$$

As before, the quantities in square brackets are molar concentrations; we have denoted by $k_{E}^{\prime}$ and $k_{Q}^{\prime}$ effective rate parameters which may actually be functions of [Q].

In a typical steady-state fluorescence experiment, the fluorescence intensity is measured as a function of quencher concentration. Since the fluorescence intensity is directly proportional to $\left[\mathrm{A}^{*}\right]$, and since $[\mathrm{A}]$ is usually so much larger than $\left[A^{*}\right]$ that its variation, as [Q] is varied, is ignorable, Eq. (2) can be shown to lead immediately to

$$
\frac{F_{0}}{F}=\left(\frac{k_{E}}{k_{E}^{\prime}}\right)\left(1+k_{Q}^{\prime} \tau[\mathrm{Q}]\right) \text {, }
$$

where $F$ and $F_{0}$ are the fluorescence intensities with and without quencher, respectively, and $\tau$ is the lifetime in the absence of quencher $\left(k_{F}+k_{N R}\right)^{-1}$. Equation (3) is completely general; it does not require a particular mechanism for the [Q] dependences of $k_{E}^{\prime}$ and $k_{Q}^{\prime}$. According to Eq. (3), a Stern-Volmer plot-i.e., a plot of $F_{0} / F$ vs [Q]-which in the classical theory of Stern and Volmer ${ }^{5}$ is expected to be linear, will actually be linear only if both $k_{E}^{\prime}$ and $k_{Q}^{\prime}$ are independent of concentration.

Explanations for nonlinear Stern-Volmer plots, which have been given in the literature, may be subdivided conconveniently into two classes by reference to Eq. (3). The recent works of Escott and Baird and of Keizer, discussed at the outset, deal with the concentration dependence of $k_{Q}^{\prime}$, which comes about, in essence, because of spatial correlations arising from the competition between fluorophores for all of the quenchers. Older explanations have emphasized the concentration dependence of $k_{E}^{\prime}$; suggestions for the mechanism of this dependence include: (i) fluorophores and quenchers form chemically distinct ground-state complexes in which any excitation is immediately extinguished $^{6,7}$; (ii) a finite-range interaction between fluorophore and quencher produces the same result as complex formation without leaving any chemical evidence for such complexes ("static quenching")"; (iii) a newly excited fluorophore with a quencher immediately adjacent has a much higher probability of being quenched than one that has to wait for a quencher to diffuse in for a collision $^{9}$; and (iv) various combinations of these. ${ }^{4,10}$

In the remainder of this paper we will assume that the absence of direct evidence for ground-state complexes or for long-range quenching, in the systems of interest, perimits us to ignore mechanisms (i) and (ii), above. We present, here, an approximate treatment of diffusionlimited fluorescence quenching in which explicit [Q] dependences of $k_{Q}^{\prime}$ and $k_{E}^{\prime}$ are found; we show that in this approximation both dependences are due to competitive renormalization and that both, in a sense, are related to mechanism (iii), above. In the next section we spell out the details of the theoretical model which provides these results. In Sec. III we compare our theory with actual quenching experiments.

\section{EXTENDED SMOLUCHOWSKI THEORY OF STEADY-STATE FLUORESCENCE QUENCHING}

\section{A. Statement of the problem}

We assume that quenching of the excited fluorophore by the impurity quencher occurs via an interaction of very short range, requiring, perhaps, the overlap of the molecular orbitals -i.e., an "encounter." When such a pair is contained within a solvent cage with no intervening solvent molecules, the pair members can make repeated encounters without undergoing diffusive motion; such a configuration defines what we mean by "in contact." The pairwise encounters of fluorophores and quenchers may be described in an average way by introducing the pair concentration $c(r, t)$, which is the number of $A^{*}-Q$ pairs per unit volume squared, at time $t$, with pair member separation $r$; for simplicity we assume spatial isotropy around each molecule. If we let $V$ be the volume defined by the solvation shell surrounding an $A^{*}-Q$ pair in contact and let $R$ be the effective radius of $V$, then the quantity $V c(R, t)$ will be the number of excited fluorophores, per unit volume, in contact with a quencher, at time $t$. Assuming that the quenching reaction proceeds at a characteristic rate $k_{0}$ (which reflects whatever activation barriers and steric hindrances that may exist) once fluorophore and quencher are in contact, then $k_{0} V c(R, t)$ represents the rate of loss of $A^{*}$ 's, per unit volume, averaged over the sample, due to collisional quenching by $Q^{\prime} s$. In our model, all of the molecular level correlations which participate in the renormalization of the sample-average kinetics enter through $c(R, t)$. We, therefore, can write a sample-average kinetics equation for the steady state, which is completely equivalent to $\mathrm{Eq}$. (2), in the form

$$
k_{E}[\mathrm{~A}]=\left(k_{F}+k_{N R}\right)\left[\mathrm{A}^{*}\right]+k_{0} V c(R) / N^{\prime} \text {, }
$$

where $N^{\prime}=6.023 \times 10^{20} \mathrm{~cm}^{-3} \mathrm{M}^{-1}$ converts molecular concentrations into molar concentrations. In this equation, the left-hand side is the total, not [as in Eq. (2)] the effective, excitation rate, and all quantities are time independent. The problem of interest, then, becomes one of determining $c(r)$ to some reasonable level of approximation. Our approximation for $c(r)$ is based on the Smoluchowski theory for coagulation, suitably generalized for our purpose and extended to account for competitive reactions. ${ }^{11-14}$ In its most general form, this model assumes that $c$ satisfies the field equation

$$
D \nabla^{2} c+\left(\partial_{t} c\right)_{\text {react }}=\partial_{t} c,
$$

where $D$ is the relative fluorophore-quencher diffusivity ${ }^{15}$ and $\left(\theta_{t} c\right)_{\text {react }}$ describes all possible sinks and sources for $A^{*}-Q$ pairs with separation $r$. To proceed we must solve the steady-state form of Eq. (5) subject to appropriate boundary conditions and a reasonable representation for $\left(\partial_{t} c\right)_{\text {react }}$. 


\section{B. Boundary conditions}

The pair concentration $c(r)$ is a measure of the coarse-grained, nonequilibrium, spatial correlations which exist between an excited fluorophore and a quencher molecule during the excitation/quenching processes. These correlations are significant for small pair-member separations, but vanish as $r$ becomes large. The pair concentration function, therefore, can be assumed to approach a sample-average value in the latter limit. If we let $\rho, \rho_{*}$, and $\rho_{\mathrm{Q}}$ be, respectively, sample-average number densities of A's, $\mathrm{A}^{*}$ 's, and Q's (i.e., $\rho=[\mathrm{A}] N^{\prime}$, and so on), we can write

$$
c(r \rightarrow \infty) \rightarrow \rho_{*} \rho_{Q},
$$

which, in turn, provides one boundary condition on Eq. (5).

A second boundary condition is obtained by considering the events which can occur to $A^{*}-Q$ pairs in contact. As we have already noted, the number density of such pairs is given by $V c(R)$. In steady state, the kinetics of such pairs can be expressed as

$$
0=V \Phi_{E}(R)-\left(1 / \tau+k_{0}\right) V c(R)+4 \pi R^{2} D \partial_{r} c(R) .
$$

The first term on the right-hand side of $\mathrm{Eq} .(7)$ is the rate of production of $A^{*}-Q$ pairs, in contact, due to excitation of an $\mathrm{A}$ with a $\mathrm{Q}$ already in contact; we will return to its form momentarily. The second term in Eq. (7) describes fluorescence and nonradiative decay and collisional quenching. The last term is the rate of change of the concentration of $A^{*}-Q$ pairs, in contact, due to relative diffusion through the solvation shell.

\section{Sinks and sources in the Waite approximation}

To complete the specification of the field equation (5) for $c$ we must write down a tractable approximation for $\left(\partial_{t} c\right)_{\text {react. }}$. In general, this term requires knowledge of the whole hierarchy of multiple particle correlations, and would depend on three-particle concentrations, fourparticle concentrations, and so on. Here, we follow the approximation invoked by Waite ${ }^{12}$ in his version of the Smoluchowski theory, namely, to write $\left(\partial_{t} c\right)_{\text {react }}$ in terms of products of pair concentrations only. In this approximation we have, for pair-member separation $r$,

$$
\left(\partial_{t} c\right)_{\text {react }}=\Phi_{E}(r)-c(r) / \tau-\left[\frac{k_{0} V c(R)}{\rho_{*}}\right] c(r) .
$$

The first term on the right-hand side of Eq. (8), similar to the first term in $\mathrm{Eq}$. (7), describes the production of $A^{*}-Q$ pairs, with member separation $r$, as the result of the excitation of A's already paired with Q's at a distance $r$. The second term results because the $A^{*}$ of each $A^{*}-Q$ pair has a finite lifetime $\tau$, even in the absence of $Q$. The third term measures the rate of disappearance of $A^{*}-Q$ pairs, of separation $r$, due to the collisional quench of $\mathrm{A}^{*}$ by another $\mathrm{Q}$; in the Waite approximation, the rate of this competitive quench of $\mathrm{A}^{*}$ is just the probable rate any $\mathrm{A}^{*}$ is quenched by collision, which can be expressed as the concentration of $A^{*}$ 's quenched by collision per unit time per unit $A^{*}$ concentration.
The excitation rate term $\Phi_{E}$ in both Eq. (7) and (8) depends on the $A-Q$ pair concentration. When an $A^{*}$ is quenched by collision with a $Q$, a close-lying $A^{*}-Q$ pair is removed from the sample [this is the origin of the spatial dependence of $c(r)]$ and replaced by a closelying $A-Q$ pair. In actual experiments, the time between successive excitations of the same fluorophore is typically much longer than the time necessary for the $A-Q$ pair distribution to relax by diffusion. Thus, the quantity $\Phi_{E}$ in both Eqs. (7) and (8) can be replaced by the spatially independent expression

$$
\Phi_{E}=k_{E} \rho \rho_{\mathrm{Q}} \text {. }
$$

Note that the form chosen for $\left(\partial_{t} c\right)_{\text {react }}$ has the appealing feature that for large $r$, as particle correlations are lost, Eq. (5) $\rightarrow$ which describes the kinetics of correlated pairs - reduces identically to the expression for the sample-average kinetics given by $\mathrm{Eq}$. (4). That is, the form chosen for $\left(\partial_{t} c\right)_{\text {react }}$ ensures that the model described here is internally consistent.

\section{Solution to the pair concentration field equation}

We are now ready to generate a formal solution to Eq. (5). A simplification results by introducing a coarse-grained $A^{*}-Q$ pair correlation function $h(r)$, through the definition

$$
c(r)=\rho_{*} \rho_{\mathrm{Q}}[1-h(r)] ;
$$

loss of correlation for large $r$ requires $h \rightarrow 0$ as $r \rightarrow \infty$. The steady-state form of Eq. (5), in terms of $h$, is then

$$
\nabla^{2} h-\beta^{2} h=0,
$$

where

$$
\beta^{2}=1 / D \tau+k_{0} V c(R) / D \rho_{*} .
$$

A formal solution to $\mathrm{Eq}$. (11), which has the proper asymptotic behavior in $r$, is

$$
h(r)=\frac{A e^{-\beta r}}{r},
$$

where $A$ is a constant of integration which is easily evaluated by reference to the boundary condition (7). Equation (13) is only a formal solution to Eq. (11), of course, because $\beta$ itself depends on $h(R)$. We will address the problem of untangling this transcendental relationship below.

\section{E. Renormalized rate constants and the Stern-Volmer relation}

Insertion of Eq. (13) into Eq. (7) through Eq. (10), allows us to find another formal expression, this time for the total collisional quenching rate:

$$
k_{0} V c(R)=4 \pi D R \phi(1+\beta R) \rho_{*} \rho_{Q}+k_{E} \phi V \rho_{Q} \rho,
$$

where

$$
\phi=k_{0} /\left[1 / \tau+k_{0}+4 \pi D R(1+\beta R) / V\right] .
$$

Again, Eq. (14) is a formal expression because $\beta$, which appears on the right-hand side, depends on $c(R)$. On the other hand, Eq. (14) could be used in conjunction with the defining relation for $\beta[\mathrm{Eq}$. (12)] to evaluate the explicit $\rho_{Q}$ dependence of $\beta$ by iteration. Thus, for the mo- 
ment at least, one may imagine that the right-hand side of $\mathrm{Eq}$. (14) is a known function of $\rho_{Q}$ (or [Q]).

If we substitute Eq. (14) into the sample-average kinetics equation (4), multiply and divide by proper factors of $N^{\prime}$ to convert to molar units, and rearrange terms to agree with the form given in $\mathrm{Eq}$. (2), we find that

$$
k_{E}^{\prime}=k_{E}\left(1-\phi V N^{\prime}[\mathrm{Q}]\right)
$$

and

$$
k_{Q}^{\prime}=4 \pi D R N^{\prime} \phi(1+\beta R) .
$$

Use of these rate parameters in Eq. (3) leads to a Stern-Volmer relation which is renormalized by fluorophore-quencher spatial correlations; it is

$$
\frac{F_{0}}{F}=\frac{1+K_{\mathrm{SV}} \phi(1+\beta R)[\mathrm{Q}]}{1-\phi V^{\prime}[\mathrm{Q}]},
$$

where $K_{\mathrm{Sv}}$, the Stern-Volmer constant, replaces $4 \pi D R \tau N^{\prime}$, and $V^{\prime}$, the encounter volume measured in $M^{-1}$, replaces $V N^{\prime}$. Note that this expression diverges as $V^{\prime}[\mathrm{Q}]-1$. Thus, the transcendental relation [Q] $=1 / \phi V^{\prime}$ ( $\phi$ is an implicit function of [Q]) defines an upper bound, in quencher concentration, on the domain of validity of the Waite approximation.

Let us examine the nature of the function $\phi$ given by Eq. (15) a little more closely. Since $V$ is $4 \pi R^{3} / 3$, the ratio $4 \pi D R / V$ which appears in $\phi$ can be rewritten as $3 D / R^{2}$, a quantity roughly equivalent to the rate at which a fluorophore and a quencher can diffuse a relative distance $R$. If we denote $3 D / R^{2}$ by $k_{D}$ and the decay rate $1 / \tau$ by $k_{L}$ ( $L$ for lifetime), then $E q$. (15) can be written as

$$
\phi=\epsilon /\left[1+(1-\epsilon) \beta R /\left(1+k_{L} / k_{D}\right)\right],
$$

where

$$
\epsilon=k_{0} /\left(k_{0}+k_{L}+k_{D}\right) .
$$

It is frequently the case in actual systems that $k_{L}$ is only a few percent of $k_{D}$. When this is so, $\epsilon$ is approximately $k_{0} /\left(k_{0}+k_{D}\right)$ and consequently has a natural interpretation: it is the probability that an $A^{*}-Q$ pair in contact will collisionally quench the excited state before the pair separates by diffusion. In other words, $\epsilon$ is a measure of the quenching efficiency of an $A^{*}-Q$ collision. When $k_{0} \gg k_{D}$, both $\epsilon$ and $\phi$ are about unity. On the other hand, when $k_{0} \ll k_{D}$, $\epsilon$ is small and $\phi \approx \epsilon /(1+\beta R)$. Furthermore, $K_{\mathrm{Sv}} / V^{\prime}=k_{D} / k_{L}$, which, as we have said, is usually large compared to unity. For a wide range of [Q] values, under these conditions, the right-hand side of Eq. (18) will be well approximated by the simple linear expression $1+\epsilon K_{\mathrm{SV}}[\mathrm{Q}]$. Stern-Volmer plots for inefficient quenchers will, the refore, be essentially linear over a wide range of [Q]. Note, however, that Eq. (18) predicts nonlinearity even for inefficient quenching at sufficiently high [Q]. Note, too, that the effective Stern-Volmer constant for inefficient quenchers is $\epsilon K_{\mathrm{SV}}$, not $K_{\mathrm{Sv}}$. Since $\epsilon \approx k_{0} / k_{D}$ in the low efficiency limit, the effective Stern-Volmer constant will be approximately equal to $k_{0} \tau V^{\prime}$.

While Eq. (18), when supplemented with an iterative evaluation of the [Q] dependence of $\beta$, provides a means for comparing the model presented here with experiment, a computationally simpler algorithm is possible. Recall the definition of $\beta$ given in Eq. (12). We can rewrite Eq. (12) as

$$
\begin{aligned}
(\beta R)^{2} & =R^{2}\left[\rho_{*} / \tau+k_{0} V c(R)\right] D \rho_{*} \\
& =R^{2} k_{E} \rho / D \rho_{*},
\end{aligned}
$$

the latter equality being a result of $\mathrm{Eq}$. (4). Since the fluorescence intensity $F$ is given by $F=\alpha k_{F} \rho_{*}$, where $\alpha$ is the "view factor" which characterizes the experimental setup, we have from Eq. (4) $k_{E} \rho=F_{0} / \alpha k_{F} \tau$. Therefore, Eq. (21) becomes

$$
\begin{aligned}
(\beta R)^{2} & =\left(R^{2} / D \tau\right)\left(F_{0} / F\right) \\
& =\left(3 k_{L} / k_{D}\right)\left(F_{0} / F\right) .
\end{aligned}
$$

For efficient quenching reactions, where $k_{L}$ is much smaller than $k_{D}$ and $\epsilon$ the order of unity, the function $\phi$ will only weakly vary as [Q] varies [see Eq. (19)]. As a consequence, if we treat $\phi$ as having a fixed value, insertion of Eq. (22) into Eq. (18) leads essentially to a quadratic equation in $F_{0} / F$ which can be solved to yield $F_{0} / F$ as a closed-form function of [Q]. The result is

$$
\frac{F_{0}}{F}=\frac{1+K_{\mathrm{SV}} \phi[\mathrm{Q}]}{1-V^{\prime} \phi[\mathrm{Q}]}+\frac{\gamma^{2} K_{\mathrm{SV}}^{2} \phi^{2}[\mathrm{Q}]^{2}+2 \gamma K_{\mathrm{SV}} \phi[\mathrm{Q}] S}{2\left(1-V^{\prime} \phi[Q]\right)^{2}},
$$

where

$$
\gamma=\left(3 k_{L} / k_{D}\right)^{1 / 2}
$$

and

$S=\left\{\left(1+K_{\mathrm{SV}} \phi[\mathrm{Q}]\right)\left(1-V^{\prime} \phi[\mathrm{Q}]\right)+\gamma^{2} K_{\mathrm{SV}}^{2} \phi^{2}[\mathrm{Q}]^{2} / 4\right\}^{1 / 2}$.

We will utilize Eq. (23) below when we compare our theory with experimental data.

\section{F. Origin of the concentration dependence of the rate parameters}

Before testing predictions of Eq. (23) against experiment, we pause to comment on the origin, in our model, of the concentration dependence of the rate parameters shown in Eqs. (16) and (17).

It is well known ${ }^{12}$ that the time-dependent solutions of Eq. (5), for the situation in which $\Phi_{E}$, in the supplementary condition (8), represents excitation by an instantaneous flash pulse [i.e. , $\left.\Phi_{E} \propto \delta(t)\right]$, show a rapid initial transient followed by a much more gradual time development. This transient behavior is related to the fact that close-lying $A^{* '}$ ' and $Q$ 's collide and quench with much higher likelihood than do A*'s and Q's which have to diffuse any appreciable distance before colliding. It produces, in turn, a rapid transient enhancement in the sample-average rate at which $A^{*}$ 's are quenched by collision with $Q$ 's.

In a steady-state experiment, where $\Phi_{E}$ is constant in time, close-lying pairs are replenished at a constant rate and the transient phenomena cited above are continuously folded into the steady-state kinetics. The resulting integrated enhancement to the steady-state collisional quenching rate has two components in our model. Part of this enhancement comes about because 
a $Q$ in contact with an $A^{*}$ at the time of the latter's excitation competes very successfully, vis-a-vis all the other Q's, for the quench of that $A^{*}$; the probability of such a quench will depend on, among other things, the excitation rate $k_{E}$ and the efficiency of quench upon collision, and is represented by the second term on the right-hand side of Eq. (14). The other part of the enhancement is a result of the overlap of the $A^{*}-$ $Q$ pair diffusion profiles (correlation functions).

Random diffusion also replenishes close-lying pairs-at a rate dependent on the relative diffusivity $D$. This contribution is included in the first term on the right-hand side of Eq. (14). Of course, it is the former enhancement which leads to the effective concentration dependence of $k_{E}^{\prime}$, the latter to the effective concentration dependence of $k_{Q}^{\prime}$.

\section{COMPARISON WITH EXPERIMENT}

We have chosen four different sets of experimental fluorescence quenching data to test the model presented above. Two of the data sets, quenching of $N$-acetyltryptophanamide (NATA) in water by iodide and acrylamide, were generated in our own laboratory while the other two, quenching of perylene in dodecane by oxygen ${ }^{16}$ and quenching of 1,2-benzanthracene in 1,2-propanediol by carbon tetrabromide, ${ }^{9}$ were taken from the literature. These fluorophore-quencher combinations were chosen because the fluorophores all exhibit single exponential fluorescence decay under the experimental conditions employed, there is no spectral evidence for ground state complex formation between fluorophore and quencher, and the Stern-Volmer plots all show substantial positive curvature. In addition, the parameters required for the application of Eq. (23) to the quenching data-namely, the intrinsic lifetimes, contact radii, and diffusivities - were either readily available or could be adequately estimated.

\section{A. Quenching of NATA}

Over the past several years, we have been interested in the effects of external quenchers, such as acrylamide and iodide ion, on the fluorescence of tryptophan derivatives. Both of these quenchers cause significant dimunition of tryptophan fluorescence when they are present in the range $0-0.5 \mathrm{M}$, and both lead to nonlinear SternVolmer plots. Quenching data for NATA specifically, with either acrylamide or iodide, can be found in the literature ${ }^{17}$ but over a more limited range of quencher concentration than we report here. We have chosen to extend the quencher concentration range for these studies to provide a more rigorous test of the proposed quenching model.

In our experiment, absorption measurements were obtained on a Beckman D.U. spectrophotometer. The maximum absorbance ( $1 \mathrm{~cm}$ cell) of NATA at the exciting wavelengths used was less than 0.07. Fluorescence measurements were obtained on a Perkin Elmer MPF-2A spectrofluorometer using $5 \mathrm{~nm}$ excitation and emission slits. Although NATA has its absorption maximum near $280 \mathrm{~nm}$, the excitation wavelengths for the quenching experiments were 295 (acrylamide) and
$290 \mathrm{~nm}$ (iodide). These excitation wavelengths were selected to minimize background absorption of the quencher which can be appreciable at the higher quencher concentrations used. Even so, the fluorescence intensities from acrylamide quenching experiments had to be corrected for residual acrylamide absorption at $295 \mathrm{~nm}$ $(a=0.23)^{17}$ using the method of Parker. ${ }^{18}$ The emission wavelength used for the quenching experiments was 354 $\mathrm{nm}$, the uncorrected wavelength of maximum fluorescence for NATA in water as measured on our fluorometer. Neither quencher causes a shift in the NATA fluorescence spectrum, so the fluorescence intensities did not have to be corrected for the wavelength dependence of the emission monochromator-photomultiplier combination.

In the acrylamide quenching experiments, a $3.00 \mathrm{ml}$ aliquot of NATA in $0.005 \mathrm{M}$ phosphate buffer, $p \mathrm{H} 6.0$, was added to a fluorescence cell and placed in the sample compartment of the MPF-2A. After the fluorescence intensity was measured, 12 separate $30 \mu \mathrm{l}$ volumes of 2. $00 \mathrm{M}$ acrylamide were added to the cell, without removing it from the sample compartment, and the fluorescence intensity was recorded after each addition. An SMI micro/pettor was used for the $30 \mu l$ additions, and the excitation shutter on the MPF-2A was closed in between measurements to minimize sample photolysis from the xenon lamp source. The measured fluorescence intensities were corrected for dilution and for acrylamide absorption, as mentioned above, before they were used in conjunction with Eq. (23).

The ionic strength of the iodide quenching solutions was held constant at $0.500 \mathrm{M}$ by the addition of 0 $1.00 \mathrm{~m} \ell$ of $5.00 \mathrm{M} \mathrm{NaCl}$ to $10 \mathrm{~m} \ell$ volumetric flasks containing a fixed concentration of NATA $\left(A_{290} \leq 0.07\right)$,

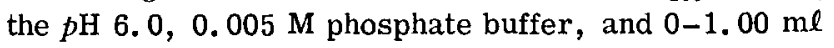
of $5.00 \mathrm{M} \mathrm{KI}$. The latter solution was freshly prepared, stored in brown bottles, and made $1 \times 10^{-4} \mathrm{M}$ in $\mathrm{Na}_{2} \mathrm{~S}_{2} \mathrm{O}_{3}$ to retard oxidation of the iodide. Nine different solutions, containing 0-0.500 M KI, were used in a given quenching experiment. Addition of peptide, buffer, $5.00 \mathrm{M} \mathrm{KI}$, and $5.00 \mathrm{M} \mathrm{KCl}$ were made using an SMI Digital Adjust micro/pettor.

The corrected fluorescence intensities from both these experiments were used to test the validity of Eq. (23). In using Eq. (23), we assumed we knew the lifetime $\tau$, the relative diffusivity $D$, and the contact raduis $R$, and attempted to fit the observed data by adjusting, effectively, the single unknown, the quenching rate constant $k_{0}$. Actually, the fitting procedure we employed was to insert $\tau, D$, and $R$ into the factors $K, V$, and $\gamma$ in Eq. (23), treat $\phi$-because of its weak dependence on [Q] (see above) - as a fixed but unknown parameter, then identify the best-fit value of $\phi$ by fitting Eq. (23) to the data with a nonlinear, least-squares algorithm. We interpret the resulting value of $\phi$ as the mean value over the range of [Q]'s used. Equation (19) shows that $\phi$ and the quenching efficiency $\epsilon$ are close in value to each other. Inserting the mean value of $\phi$ into Eq. (19) allows us to calculate a range of $\epsilon$ values, for the different values of [Q] used, all of which are compatible with the fit obtained. This range is very narrow: the 
TABLE I. Molecular parameters of the systems studied in Sec. III.

\begin{tabular}{lllccccc}
\hline \hline Fluorophore & Quencher & \multicolumn{1}{c}{ Solvent } & $T\left({ }^{\circ} \mathrm{C}\right)$ & $D\left(\mathrm{~cm}^{2} / \mathrm{s}\right) \times 10^{-5}$ & $R(\AA)$ & $\tau(\mathrm{ns})$ & Quenching efficiency, $\epsilon$ \\
\hline NATA & Acrylamide & Water & 25 & 1.5 & 6.5 & 2.8 & $0.73 \pm 0.03$ \\
NATA & $\mathrm{I}^{-}$ & Water & 25 & 1.5 & 6.2 & 2.8 & $0.40 \pm 0.04$ \\
Perylene & $\mathrm{O}_{2}$ & Dodecane & 25 & 7.8 & 5.6 & 5.4 & $0.64 \pm 0.02$ \\
1,2-Benzanthracene & $\mathrm{CBr}_{4}$ & 1,2-Propanediol & 15 & 0.045 & 7.4 & 39.2 & 1.00 \\
1,2-Benzanthracene & $\mathrm{CBr}_{4}$ & 1,2-Propanediol & 25 & 0.085 & 7.4 & 38.5 & $0.96 \pm 0.01$ \\
1,2-Benzanthracene & $\mathrm{CBr}_{4}$ & 1,2-Propanediol & 35 & 0.15 & 7.4 & 37.7 & $0.92 \pm 0.01$ \\
\hline \hline
\end{tabular}

${ }^{2}$ Ionic strength held constant at $0.5 \mathrm{M}$ by the addition of $\mathrm{NaCl}$.

procedure outlined is thus a single parameter, nonlinear fit with the fitting parameter, in essence, being $\epsilon$. We could of course, work back from $\epsilon$ through Eq. (20) to find $k_{0}$, if that were desirable.

The intrinsic lifetime of NATA in water has been measured by several groups ${ }^{19-21}$; we took its value to be 2.8 ns. (See Table I for a summary of molecular parameters used in this paper.) Space-filling models of NATA and acrylamide suggest effective van der Waals radii of about 4.0 and $2.5 \AA$, respectively; the ionic radius of $I^{-}$is known to be $2.2 \AA$. The contact radii for these systems were taken to be the sums of these radii: $R=6.5 \AA$ for NATA-acrylamide and $R=6.2 \AA$ for NATAI*. Relative diffusivities for these systems were approximated from the Stokes-Einstein relation using the radii cited above; we took $D=1.5 \times 10^{-5} \mathrm{~cm}^{2} / \mathrm{s}$ for both systems, recognizing that an error by a factor of as much as 2 might be involved. The agreement between the predictions of Eq. (23) and experiment is shown in Fig. 1. The largest discrepancy between theory and experiment, for any data point, is about $3 \%$; the statistical analysis of the fitting routine shows that the un-

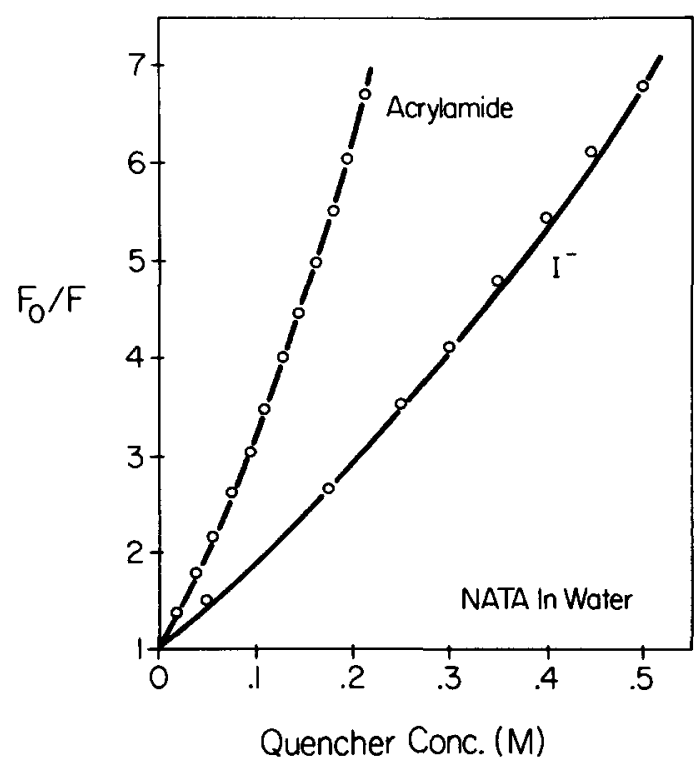

FIG. 1. Stern-Volmer plot for the quenching of the fluorophore NATA in water, at $25^{\circ} \mathrm{C}$, by the quenchers iodide ion and acrylamide. The circles are actual data, the solid curves are the predictions of Eq. (23) using the molecular parameters described in the text. Error bars are about the size of the circles shown. certainties associated with the fit (the standard deviations in the best values of $\phi$ ) are less than $0.6 \%$. For our choices of molecular parameters, we find the quenching efficiency for a collision between NATA and acrylamide to be about $75 \%$, and for NATA and I- about $40 \%$, in water at $25^{\circ} \mathrm{C}$.

\section{B. Other experiments}

In addition to the NATA fluorescence experiments described above, we have also examined data published by other investigators. By way of example, we discuss two studies where molecular radii and diffusivities can be estimated with some confidence.

First, we look at the quenching of perylene by oxygen, in the solvent dodecane, examined by Lakowicz and Weber. ${ }^{16}$ These authors measured $\tau$ to be $5.4 \mathrm{~ns}$. Again, space-filling models suggest that a reasonable contact radius for perylene $-\mathrm{O}_{2}$ is about $5.6 \AA$. Oxygen is known to be a rapid diffuser in liquids and its diffusivity is non-Stokesian. Ware ${ }^{22}$ has measured the diffusivities of perylene and $\mathrm{O}_{2}$ in hydrocarbon solvents

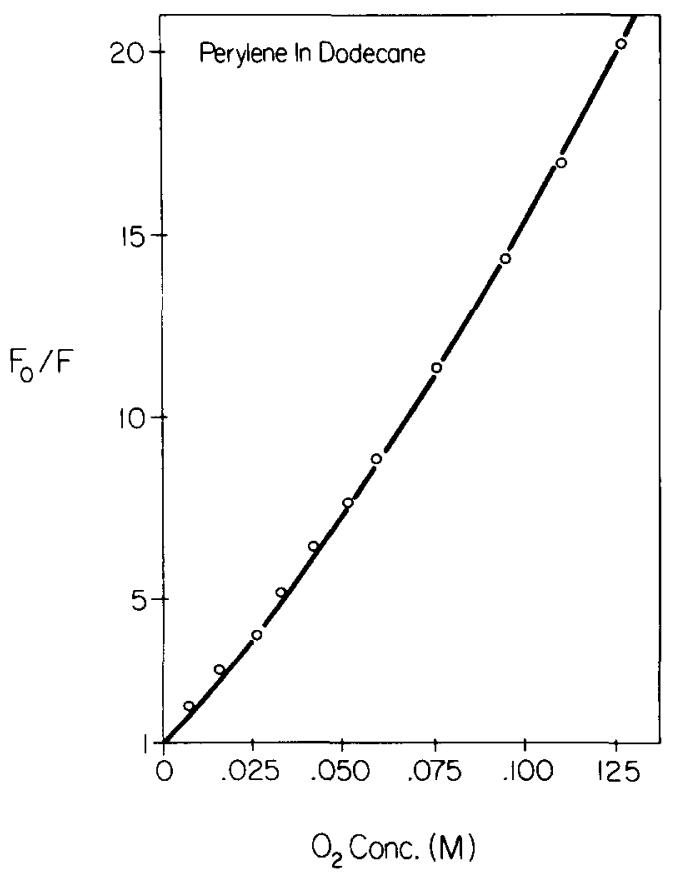

FIG. 2. Stern-Volmer plot for the quenching of perylene in the solvent dodecane, at $25^{\circ} \mathrm{C}$, by molecular oxygen (data points taken from Lakowicz and Weber, Ref. 16). The solid curve, again, is the prediction of Eq. (23). 


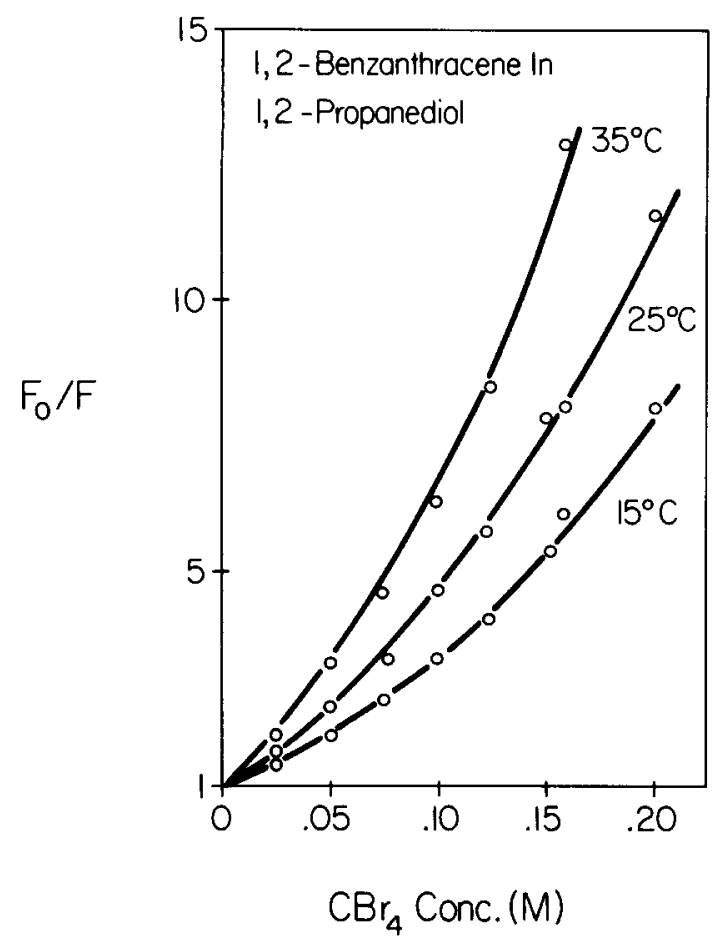

FIG. 3. Stern-Volmer plot for the quenching of 1,2-benzanthracene in the solvent 1,2-propanediol, at 15,25 , and $35^{\circ} \mathrm{C}$, by carbon tetrabromide (data points taken from Nemzek and Ware, Ref. 9).

similar to dodecane; we use these results to estimate $D$ as $7.8 \times 10^{-5} \mathrm{~cm}^{2} / \mathrm{s}$. With these values Eq. (23) leads to the fit of the data of Lakowicz and Weber shown in Fig. 2. Again, the fit has a very small statistical uncertainty $(\leq 0,3 \%)$; it leads to the estimate that collisions between perylene and $\mathrm{O}_{2}$, in dodecane at $25^{\circ} \mathrm{C}$, quench about $65 \%$ of the time.

A final example is provided by the study of the quenching of 1,2-benzanthracene by carbon tetrabromide in the solvent 1,2-propanediol, done by Nemzek and Ware. ${ }^{\circ}$ They have measured $F_{0} / F$ as a function of $\left[\mathrm{CBr}_{4}\right]$ for three different temperatures, 15,25 , and $35^{\circ} \mathrm{C}$. They report $\tau$ values of $39.2,38.5$, and $37.7 \mathrm{~ns}$, respectively, for these temperatures. The molecule 1,2-benzanthracene is an elongated, planar structure whose longest linear dimension is slightly over $9 \AA$. Since diffusional collision with an elongated molecule occurs almost as frequently as with a sphere of diameter equal to the length of the longest axis, ${ }^{23}$ we take the van der Waals "radius" of 1,2-benzanthracene to be about $4.5 \AA$. Using $2.9 \AA$ for the van der Waals radius of $\mathrm{CBr}_{4}$, we have $R=7.4 \AA$. Measurements of diffusivities in 1,2propanediol at $25^{\circ} \mathrm{C}^{24}$ indicate the relative diffusivity for this system to be about $8.5 \times 10^{-7} \mathrm{~cm}^{2} / \mathrm{s}$, at this temperature. Using an activation energy for diffusion in 1,2-propanediol of $45 \mathrm{~kJ} / \mathrm{mol},{ }^{24}$ we estimate the relative diffusivities to be $4.5 \times 10^{-7} \mathrm{~cm}^{2} / \mathrm{s}$ at $15^{\circ} \mathrm{C}$ and 1.5 $\times 10^{-6} \mathrm{~cm}^{2} / \mathrm{s}$ at $35^{\circ} \mathrm{C}$. The data shown in Fig. 3 demonstrate somewhat more scatter than those in the previous figures. Nonetheless, Eq. (23) fits the data with no discrepancy larger than $5 \%$. We see that the effi- ciency of quench of a collision between 1,2-benzathracene and $\mathrm{CBr}_{4}$ is nearly $100 \%$ in 1,2-propanediol over the temperature range of this study.

\section{Summary}

In this paper, we have presented a theoretical model to describe the collisional quenching of excited fluorophores by impurity quenchers in a dense medium which accounts for competition among reactants. We have shown that these competitive processes lead to effective concentration dependences for both the bulk quenching and excitation rate parameters. We fit our model to a variety of experimental data by adjusting a single free parameter-the efficiency of quenching by a fluorophorequencher collision $\rightarrow$ and, in all cases examined, obtained excellent agreement.

\section{ACKNOWLEDGMENTS}

We would like to thank Professor Joel Keizer for sharing with us a preprint of his work. This research was supported, in part, by a grant from the Research Corporation.

${ }^{1}$ H. L. Frisch and F. C. Collins, J. Chem. Phys. 20, 1797 (1952); B. U. Felderhof and J. M. Deutch, ibid. 64, 4551 (1976); D. Peak, K. Pearlman, and P. J. Wantuck, ibid. 65, 5538 (1976); J. R. Lebehaft and R. Kapral, J. Stat. Phys. 20, 25 (1979); M. Bixon and R. Zwanzig, J. Chem. Phys. 75, 2334 (1981); M. Muthukumar and R. I. Cukier, J. Stat. Phys. 26, 453 (1981); J. Keizer, J. Phys. Chem. 85, 940 (1981); M. Muthukumar, J. Chem. Phys. 76, 2667 (1982); T. Kirkpatrick, ibid. 76, 4255 (1982); B. U. Felderhof, J. M. Deutch, and U. M. Titulaer, ibid. 76, 4178 (1982); M. Tokuyama and R. I. Cukier, ibid. 76, 6206 (1982); R. I. Cukier, J. Stat. Phys. 30, 383 (1983).

${ }^{2}$ J. K. Baird and S. P. Escott, J. Chem. Phys. 74, 6993 (1981).

${ }^{3}$ J. Keizer, J. Am. Chem. Soc. 105, 1494 (1983).

${ }^{4}$ The review by M. R. Eftink and C. A. Ghiron, Anal. Biochem. 114,199 (1981) contains many relevant references.

${ }^{5} \mathrm{O}$. Stern and M. Volmer, Phys. Z 20, 183 (1919).

${ }^{6} \mathrm{H}$. Boaz and G. K. Rollefson, J. Am. Chem. Soc. 72, 3435 (1950).

${ }^{7}$ E. J. Bowen and W. S. Metcalf, Proc. R. Soc. London Ser. A 206, 437 (1951).

8. M. Frank and S. J. Vavilov, Z. Phys, 69, 100 (1931).

${ }^{9}$ T. L. Nemzek and W. R. Ware, J. Chem. Phys. 62, 477 (1975).

${ }^{10} \mathrm{~J}$. C. Andre, M. Niclause, and W. R. Ware, Chem. Phys. 28, 371 (1978).

${ }^{11}$ M. V. Smoluchowski, Z. Phys. Chem. 92, 129 (1917).

${ }^{12} \mathrm{~T}$. R. Waite, Phys. Rev. 107, 463 (1957).

${ }^{13} \mathrm{~J}$. Yguerabide, M. A. Dillon, and M. Burton, J. Chem. Phys. 40, 3040 (1964).

${ }^{14}$ G. Wilemski and M. Fixman, J. Chem. Phys. 58, 4009 (1973).

${ }^{15}$ In general, $D$ may be a function of [A], [Q], and of $r$; see, for example, reference in D. Peak, J. Chem. Phys. 76, 3792 (1982). We approximate $D$, here, as simply the sum of the constant fluorophore and quencher diffusivities in the solvent of interest.

${ }^{16} \mathrm{~J}$. R. Lakowicz and G. Weber, Biochemistry 12, 4161 (1973).

${ }^{17}$ M. R. Eftink and C. A. Ghiron, J. Phys. Chem. 80, 486 (1976).

${ }^{18} \mathrm{C}$. A. Parker, Photoluminescence of Solutions (Elsevier, 
New York, 1968), pp. 220-222.

${ }^{19} \mathrm{~A}$. Grunwald and I. Z. Steinberg, Biochem. Biophys. Acta 427, 663 (1976).

${ }^{20}$ T. C. Werner and L. S. Forster, Photochem. Photobiol. 29, 905 (1979).

${ }^{21}$ A. G. Szabo and D. M. Rayner, J. Am. Chem. Soc. 102,
554 (1980).

${ }^{22}$ W. R. Ware, J. Phys. Chem. 66, 455 (1961).

${ }^{23}$ P. H. Richter and M. Eigen, Biophys. Chem. 2, 255 (1974).

${ }^{24}$ A. D. Osborne, H. J. V. Terrell, and M. Zaman, Trans.

Faraday Soc. 60, 395 (1964); M. Mitchell and H. J. V.

Terrell, J. Chem. Soc. Faraday Trans. 2 68, 385 (1972). 